\title{
Percepção de Saúde de Idosos Participantes de um Programa de Inclusão Digital
}

\author{
Health Perception of Elderly Participants in a Digital Inclusion Program
}

\section{RESUMO}

Objetivo: Esta pesquisa teve como objetivo investigar a percepção subjetiva de saúde de idosos, participantes de um programa de inclusão digital, no projeto Viver a Vida da Paranaprevidência. Metodologia: Fizeram parte da pesquisa 21 idosos com idade igual ou superior a 60 anos, que participaram de uma intervenção de inclusão digital no programa Viver a Vida, o qual foi criado a partir da parceria entre PARANAPREVIDÊNCIA e a Secretaria de Estado da Administração e Previdência e o Governo do Paraná. Para avaliar a percepção de saúde foi feita uma pergunta "O que é ter saúde para você?" A análise dos dados descritivos foi realizada por meio de média, desvio padrão e frequência. A análise de dados qualitativos foi feita por meio de análise temática, sendo realizada uma análise da coocorrência das palavras significativas. Na sequência da metodologia, foi aplicada uma técnica de identificação de temas chamada nuvem de palavras, utilizando o software IRaMuTeQ (Interface de R pour les analyses multidimensionnelles de textes et de questionnaires). Resultados: A nuvem de palavras, deixa claro que, na perspectiva das participantes, saúde é vida, saúde é viver bem, saúde é não ser dependente de outras pessoas e ter autonomia nas suas escolhas, não tomar medicamento, comer com qualidade, ter bom relacionamento com os outros e consigo mesmo, levando a um bem-estar físico e emocional. Conclusão: Verifica-se que o termo saúde estabelece relações com o bem-estar, emocional, viver, mental, cuidar, funcionar, comer, doença, sentir e corpo, levando a crer que a percepção de saúde para as participantes envolve todos esses fatores e não está relacionado apenas a ausência de doença.

\section{DESCRITORES}

Inclusão Digital. Saúde. Idosas.

\begin{abstract}
Objective: This research aimed to investigate the subjective perception of health of the elderly, participating in a digital inclusion program, in the project "Viver a Vida" from Paranaprevidência. Methodology: The study included 21 elderly people aged 60 years or over, who participated in a digital inclusion intervention in the Viver a Vida program that was created from the partnership between PARANAPREVIDÊNCIA and the State Secretariat for Administration and Welfare and the Government of Paraná. To assess the perception of health, the question "To you, what does it mean to have health?" was asked. The analysis of the descriptive data was performed by means, standard deviation and frequency. The analysis of qualitative data was done through thematic analysis, with an analysis of the co-occurrence of significant words. Following the methodology, a theme identification technique called Word Cloud was applied, using the IRaMuTeQ software (R Interface for the Multidimensional Analyzes by Textes and Questionnaires). Results: The word cloud makes it clear that, from the perspective of the participants, health is life, health is living well, health is not being dependent on other people and having autonomy in their choices, not taking medication, eating with quality, having good relationships with others and with oneself, leading to a physical and emotional well-being. Conclusion: It appears that the term "health" establishes relationships with "well-being", "emotional", "living", "mental", "taking care", "functioning", "eating", "illness", " feel" and "body", leading to believe that the perception of health for the participants involves all these factors and is not related only to the absence of disease.
\end{abstract}

\section{DESCRIPTORS}

Digital Inclusion. Health. Elderly.

${ }^{1}$ Acadêmica de Musicoterapia da Universidade Estadual do Paraná, Campus II de Curitiba, Curitiba, Paraná, Brasil.

${ }^{2}$ Doutorando em Educação - PPGE/UFPR. Bolsista do CNPq. Universidade Federal do Paraná - UFPR, Curitiba, Paraná, Brasil.

${ }_{3}$ Professora do Curso de Musicoterapia da Universidade Estadual do Paraná, Campus II de Curitiba e Doutoranda do Programa de Pós-Graduação em Educação da Universidade Federal do Paraná, Curitiba, Paraná, Brasil.

${ }^{4}$ Professora do Curso de Musicoterapia da Universidade Estadual do Paraná, Campus II de Curitiba e Doutoranda do Programa de Pós-Graduação em Educação da Universidade Federal do Paraná, Curitiba, Paraná, Brasil.

${ }^{5}$ Professor Doutor do Programa de Pós-Graduação em Educação PPGE/UFPR da Universidade Federal do Paraná - UFPR, Curitiba, Paraná, Brasil.

${ }^{6}$ Professora Doutora do Programa de Pós-Graduação em Educação PPGE/UFPR da Universidade Federal do Paraná - UFPR e Docente do Curso de Musicoterapia da Universidade Estadual do Paraná, Campus II de Curitiba, Curitiba, Paraná, Brasil. 
A expectativa de vida humana vem aumentando consideravelmente, não só no Brasil, mas no mundo ${ }^{1}$. Segundo dados do Instituto Brasileiro de Geografia e Estatística - IBGE, o envelhecimento populacional está inversamente proporcional ao crescimento das demais faixas etárias. Em 2004 , os idosos representavam $9,7 \%$ da população brasileira, enquanto, pessoas de 0 até 29 anos eram $54,4 \%$ e em 2060 , a previsão é de que a população idosa será de 33,7\% contra $28,3 \%$ do outro grupo ${ }^{1}$.

Ao longo da vida ocorrem mudanças no cérebro, variando de pessoa para pessoa, e entre os sexos. A partir dos 30 anos, dá-se início uma redução do tamanho cerebral e, ainda que comece lentamente, em geral, ao chegar próximo aos 90 anos, o cérebro já perdeu em torno de $10 \%$ de seu peso. Inicialmente, este fato estava relacionado à perda de neurônios no córtex cerebral (em torno de 50.000 por dia, o que no final da vida representa em torno de $3 \%$ do total de original de neurônios), responsável pelas funções cognitivas, mas novas pesquisas atribuem esta redução do peso cerebral a uma redução no tamanho das células nervosas devido à perda de axônios, dendritos e sinapses no córtex frontal, área do cérebro responsável pelas funções cognitivas de alto nível ${ }^{2,3}$.

O envelhecimento seguidamente é confundido com longevidade ou expectativa de vida. Convém esclarecer que o envelhecimento é um conjunto de processos ou condições de saúde que progressivamente evoluem para o decréscimo nas funções biológicas e psíquicas. A saúde biológica e a saúde autorrelatada são avaliadas de forma diferente, mas ambos são preditores de condições de bem estar na idade idosa ${ }^{4}$.

A Organização Mundial da Saúde OMS inclui na definição de saúde, o bem-estar social entre os indivíduos, que pode ser determinado pela própria biologia humana, pelo ambiente físico, social e econômico, pelo estilo de vida, por hábitos alimentícios, pela prática de exercícios físicos, pelo bem-estar emocional e outros fatores determinantes para ao aumento da qualidade de vida e um estado de saúde equilibrado ${ }^{5}$. Geralmente, saúde e qualidade de vida são dois temas muito relacionados, uma vez que a saúde contribui para melhorar a qualidade de vida e esta é fundamental para que um indivíduo ou comunidade tenha saúde. Com isso, os indivíduos têm se preocupado com esses fatores relacionados à saúde e doença e tem buscado bem-estar que possa gerar melhor qualidade de vida.

Para Nemes e Vagetti ${ }^{6}$ a promoção da saúde apresenta-se como uma estratégia promissora para enfrentar os múltiplos problemas de saúde e deve estar associada a um conjunto de valores e uma combinação de estratégias girando em torno da ideia de responsabilidade múltipla, que está relacionado com a qualidade de vida. $A$ inclusão digital pode ser um importante meio nesse processo ${ }^{6}$.

A inclusão digital, proporcionada pela aprendizagem do uso de redes sociais virtuais, é considerada um fator determinante para a manutenção e/ou melhoria da qualidade de vida dos idosos, podendo proporcionar a esta população uma maior autonomia dos efeitos negativos para aqueles que advêm de experiências de vida difíceis ${ }^{7,8}$.

Os grupos de idosos possibilitam uma 
interatividade social mais intensa, proporcionando a troca de experiências e de atividades educacionais, que promovem a formação e a educação continuada, além das oficinas de informática que melhoram a qualidade de vida, pois auxiliam na interatividade em uma experiência social que afasta o estigma do isolamento ${ }^{9,10}$.

Segundo Silveira ${ }^{10}$, em um estudo visando a inclusão digital realizados com 92 idosos em Porto Alegre/RS, a inclusão digital desperta muito interesse entre os idosos, principalmente devido à percepção que o aprendizado de informática pode ser benéfico para a sua saúde. "[...] As oficinas de informática favoreceram não só a inclusão no meio digital, mas também o convívio com outras pessoas das mais diversas faixas etárias, levando o indivíduo idoso a melhorar sua autoestima, seu humor e evitando o isolamento social".

A inclusão digital contribui para a redução do isolamento social, para a melhoria dos processos neurocognitivos e para a qualidade de vida dos idosos, principalmente por meio de processos rápidos de comunicação nas relações interpessoais com os grupos formais e informais e, também, transgeracionais ${ }^{10}$.

Nesse sentido, este estudo teve como objetivo investigar a percepção subjetiva do termo saúde para os idosos participantes de uma intervenção de inclusão digital no programa Viver a Vida da Paranaprevidência.

\section{METODOLOGIA}

Este estudo caracteriza-se como uma abordagem qualitativa. Para a caracterização da amostra foi realizada a média, o desvio-pa- drão, o valor mínimo e máximo, a frequência absoluta e relativa dos dados sociodemográfico e econômicos.

Fizeram parte desse estudo vinte e um idosos servidores aposentados do Estado do Paraná, com idade igual ou superior a 60 anos, que participaram de uma intervenção de inclusão digital no programa Viver a Vida, o qual foi criado a partir da parceria entre PARANAPREVIDÊNCIA e a Secretaria de Estado da Administração e Previdência e o Governo do Paraná. Todos os participantes assinaram o Termo de consentimento livre e esclarecido.

Instrumentos de coleta de dados:

Questionários Sociodemográfico

Apresenta onze questões abertas e fechadas, relacionadas com os dados de identificação e as características sociodemográficas de idosos, como nível de escolaridade, ocupação, remuneração, saúde medicamentos utilizados. ${ }^{11}$

Questionário Econômico:

Conforme o critério de Classificação Econômica do Brasil, contém informações sobre os bens possuídos e a escolaridade do responsável financeiro da residência. Categoriza-se as classes econômicas da seguinte forma: A (45 - 100 pontos); B1 (38 - 44 pontos); B2 (29 - 37 pontos); C2 (17 - 22 pontos); D (8 - 16 pontos); ou $\mathrm{E}(0-7 \text { pontos })^{12}$.

- Dados qualitativos:

Foi realizada uma pergunta para as idosas: O que é ter saúde para você? 
Procedimento de coleta de dados

Para a realização da coleta de dados, primeiramente, foi agendada uma reunião com o coordenador do Programa Viver a Vida para apresentar os objetivos e a relevância do estudo, bem como, para os esclarecimentos de possíveis dúvidas. Depois de autorizada pela coordenação, foi agendada a coleta de dados.

$\mathrm{Na}$ semana de coleta dos dados, os idosos receberam as instruções necessárias para a participação no estudo e o Termo de consentimento livre e esclarecido. Neste termo constava uma breve explicação do objetivo da pesquisa e os métodos que foram empregados. Não existiu identificação nominal dos participantes da pesquisa e, sim uma numeração e eles poderiam abandonar as avaliações a qualquer momento que desejassem.

Descrição da Intervenção de inclusão digital

Os idosos, participantes da pesquisa, fizeram parte de uma intervenção de inclusão digital, com 10 encontros de 2 horas de duração, ofertada em módulos no programa Viver a Vida da Paranaprevidência.

\section{Análise dos dados}

Os dados foram analisados a partir da estatística descritiva, mediante a frequência de ocorrências e percentuais relativos e absolutos. Foi realizada a seleção de palavras significativas que se repetiram, a separação de frases e a aproximação das falas, criando categorias. A técnica utilizada foi de análise temática, em que a noção de tema está ligada a uma afirmação a respeito de dado assunto.
Essa análise comporta um feixe de relações e pode ser representada por meio de uma palavra ou frase ${ }^{13}$.

Os dados coletados na pesquisa foram organizados e foi atribuída uma numeração por resposta e uma sequência alfabética para melhor identificação dos participantes. Seguindo as etapas propostas pela metodologia de $\operatorname{Bardin}^{13}$, foi realizada uma leitura flutuante (leitura pelo software Iramuteq do corpo textual, estabelecendo um tema, sentidos e significados das palavras), para uma primeira verificação dos significados e a descoberta da categorização a ser utilizada.

$\mathrm{Na}$ etapa seguinte, foi realizada uma análise da coocorrência das palavras significativas, momento em que foram escolhidos os temas principais do corpus textual. É possível dizer que as palavras trazem um significado que vai além da estrutura ortográfica, possuindo, intrinsecamente, um conteúdo a ser relacionado ao contexto interno e externo do participante.

Para testar estas hipóteses, foi feita a relação entre os significados das expressões e o seu contexto. Na sequência da metodologia, foi aplicada uma técnica de identificação de temas chamada de nuvem de palavras (word clouds, nuvem de tags ou nuvem de etiquetas), que se trata de um método heurístico de análise textual. Nesta ferramenta foram inseridas as respostas dos participantes à pergunta "O que é saúde para você?".

Neste mesmo processo de geração de modelos de análise, com o uso de ferramentas computacionais, foi utilizado o software IRaMuTeQ® versão 0.7 Alpha 2, uma interface gráfica para a análise de dados do software estatístico R. Foi utilizado o software para 
geração de estruturas para complementação da análise de dados. As relações observadas foram organizadas nos quadros 1 e 2 para uma melhor compreensão.

Ética na pesquisa

A participação nesse estudo foi voluntária e todos os participantes receberam uma carta de apresentação junto ao Termo de consentimento livre e esclarecido. Foi garantida a privacidade e a confiabilidade do uso das informações utilizadas no estudo, seguindo todas as exigências da Resolução no. 466/2012, do Conselho Nacional de Saúde - CNS. O projeto foi enviado ao Comitê de Ética da UNESPAR, Curitiba, Campus II, tendo sido aprovado por meio do Parecer $\mathrm{n}^{\circ}$. 2.445.838 e CAAE: 79664617.7.0000.0094.

\section{RESULTADOS E DISCUSSÃO}

O presente estudo contou com 21 participantes com idade média de 70,64ะ6,54 anos, apresentou uma maior incidência de naturalidade do estado do Paraná $(57,10 \%)$, nacionalidade brasileira $(90,50 \%)$, sexo feminino $(81 \%)$, faixa etária de 60 a 69 anos $(42,90 \%)$, cor branca $(85,70 \%)$, de estado civil casadas(os) $(42,90 \%)$, pós-graduadas(os) (52,40\%), aposentadas(os) (90,50\%), professores $(81 \%)$ e de classe social B1 $(38,10 \%)$.

O Quadro 1 e 2 apresentam os resultados oriundos de uma primeira leitura livre, flutuante $^{13}$ (leitura pelo software IRaMuTeQ do corpo textual, estabelecendo um tema, sentidos e significados das palavras), a qual levou a formulação de hipóteses, a relação entre os temas e os domínios. A resposta de cada idoso, conduz a hipótese de que se relacionam os temas com os domínios, para se chegar a um conceito. Para o tema "cuidado" verifica-se uma maior relação com os domínios "físico", "emocional" e "psicológico"; no tema "bem-estar" encontra-se maior prevalência nos domínios "físico", "emocional" e "psicológico"; nos temas "atividade física" e "alimentar" o domínio que se sobressaiu foi o "físico".

Sob o ponto de vista dos idosos, percebeu-se um tom de muita certeza do que é saúde para cada um deles. Como pode-se observar o participante 19 de forma objetiva: "saúde pra mim é um bem-estar físico psicológico". O participante 20 também afirma "saúde pra mim é um bem-estar geral, tanto físico quanto emocional". O participante 16 ressalta uma determinada condição: "Saúde pra mim é a saúde física e mental. Às vezes eu chego a achar que a saúde mental é mais importante que a física, porque se você perde o juízo não adianta você nem ter saúde física. Então, acho que a gente tem que cuidar direitinho do corpo e muito, muito mais da mente, é isso só." Já para o participante 8 diz "Saúde é estar fisicamente muito bem".

É expressivo o conceito de saúde para o participante 11 e engloba os aspectos citados acima: "Saúde da mesma forma é a busca deste equilíbrio entre o emocional, o físico e o espiritual. Buscar, já na aposentadoria, atividades que nos realizem, que nos tragam afeto e que também possamos expressar nossos sentimentos, afinal é para isso que nós fomos construídos, ou seja, ter um objetivo de vida em cada fase que vivemos, ir atrás destes objetivos tendo consciência do 
Tabela 1. Caracterização dos participantes do estudo

\begin{tabular}{|c|c|c|}
\hline Variáveis Categóricas & $n=21$ & $\%$ \\
\hline \multicolumn{3}{|l|}{ Naturalidade } \\
\hline PR & 12 & 57,10 \\
\hline SP & 04 & 19,00 \\
\hline RS & 01 & 4,80 \\
\hline MS & 01 & 4,80 \\
\hline SC & 01 & 4,80 \\
\hline Estrangeiro & 02 & 9,50 \\
\hline \multicolumn{3}{|l|}{ Nacionalidade } \\
\hline Brasileiro & 19 & 90,50 \\
\hline Estrangeiro & 02 & 9,50 \\
\hline \multicolumn{3}{|l|}{ Sexo } \\
\hline Feminino & 17 & 81,00 \\
\hline Masculino & 04 & 19,00 \\
\hline \multicolumn{3}{|l|}{ Faixa Etária (anos) } \\
\hline de 60 a 69 & 09 & 42,90 \\
\hline de 70 a 79 & 08 & 38,10 \\
\hline de 80 a 89 & 04 & 19,00 \\
\hline \multicolumn{3}{|l|}{ Cor } \\
\hline Branca & 18 & 85,70 \\
\hline Preta & 01 & 4,80 \\
\hline Amarela & 01 & 4,80 \\
\hline Parda & 01 & 4,80 \\
\hline \multicolumn{3}{|l|}{ Estado Civil } \\
\hline Solteira(o) & 04 & 19,00 \\
\hline Casada(o) & 09 & 42,90 \\
\hline Separada(o) & 05 & 23,80 \\
\hline Viúva(o) & 03 & 14,30 \\
\hline \multicolumn{3}{|l|}{ Escolaridade (completa) } \\
\hline Ensino Fundamental & 01 & 4,80 \\
\hline Ensino Médio & 03 & 14,30 \\
\hline Curso Superior & 06 & 28,60 \\
\hline Pôs Graduação & 11 & 52,40 \\
\hline \multicolumn{3}{|l|}{ Ocupação Atual } \\
\hline Aposentada(o) & 19 & 90,50 \\
\hline Pensionista & - & 0,00 \\
\hline Autônoma(o) & 02 & 9,50 \\
\hline Dona(o) de casa & - & 0,00 \\
\hline Outro & - & 0,00 \\
\hline \multicolumn{3}{|l|}{ Profissão Anterior } \\
\hline Professor(a) & 17 & 81,00 \\
\hline Administrativa(o) & 01 & 4,80 \\
\hline Bancária(o) & 01 & 4,80 \\
\hline Profissional liberal & 01 & 4,80 \\
\hline Func. Pública(o) & 01 & 4,80 \\
\hline Dona(o) de casa & - & 0,00 \\
\hline \multicolumn{3}{|l|}{ Classe Social } \\
\hline$A$ & 04 & 19,00 \\
\hline B1 & 08 & 38,10 \\
\hline B2 & 03 & 14,30 \\
\hline C1 & 05 & 23,80 \\
\hline $\mathrm{C} 2$ & 01 & 4,80 \\
\hline TOTAL & 21 & 100,00 \\
\hline
\end{tabular}

Categoriza-se as classes econômicas da seguinte forma: A (45 100 pontos); B1 (38 - 44 pontos); B2 (29 - 37 pontos); C2 (17 22 pontos); $\mathrm{D}$ ( $8-16$ pontos); ou $\mathrm{E}(0-7$ pontos). 


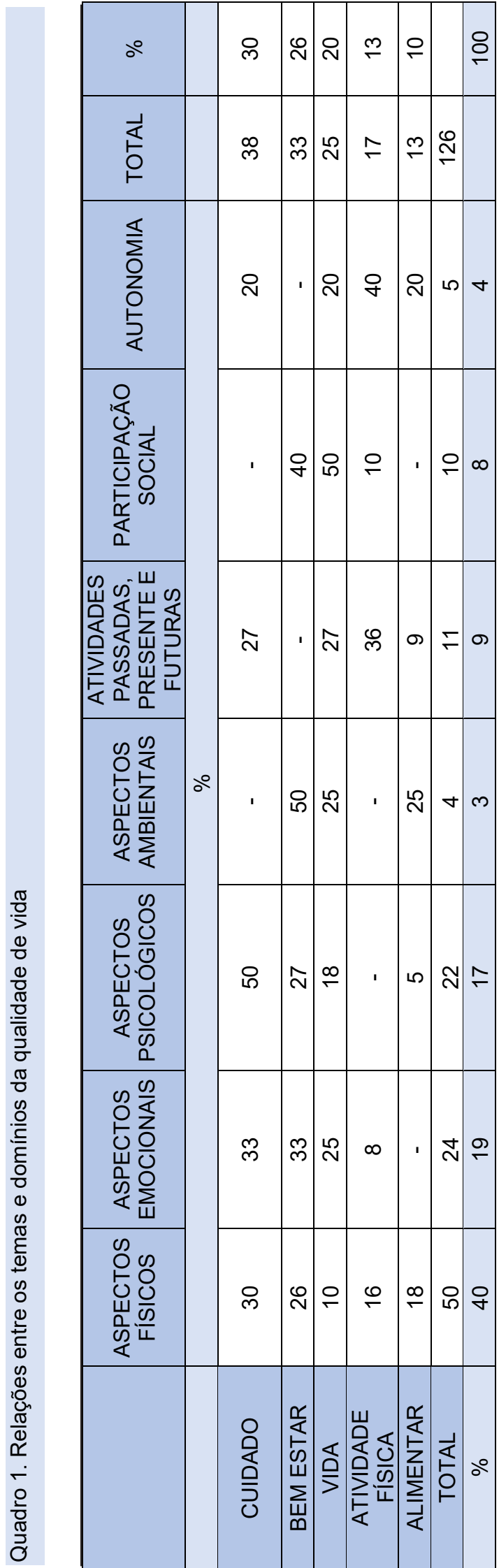




\begin{tabular}{|c|c|c|c|c|c|c|c|c|}
\hline & $\circ$ & ిల్ల & $\stackrel{\leftrightarrow}{N}$ & $\stackrel{\sim}{\text { D }}$ & $\stackrel{m}{\leftarrow}$ & 움 & & \\
\hline & 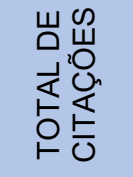 & $\stackrel{\infty}{\infty}$ & m & 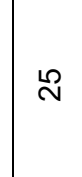 & 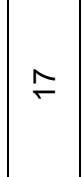 & $\stackrel{m}{\square}$ & $\stackrel{\stackrel{\leftrightarrow}{\sim}}{\sim}$ & \\
\hline & 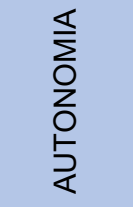 & $\widetilde{\delta}$ & & ষ & & $\stackrel{\circ}{\square}$ & 10 & ब \\
\hline & 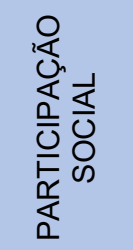 & & 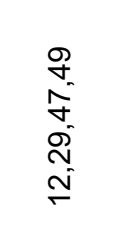 & $\begin{array}{l}\stackrel{\infty}{+} \\
\hat{m} \\
0 \\
0 \\
m \\
m \\
m \\
m\end{array}$ & 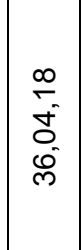 & & 우 & $\infty$ \\
\hline ¿े & 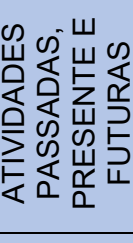 & $\begin{array}{l}\infty \\
+ \\
0 \\
0 \\
\infty \\
\infty\end{array}$ & & $\begin{array}{l}\text { ले } \\
\text { m. } \\
\stackrel{m}{m}\end{array}$ & $\begin{array}{l}\infty \\
0 \\
0 \\
m \\
\infty \\
\infty \\
\infty \\
5\end{array}$ & $\mathscr{q}$ & $\mp$ & $\sigma$ \\
\hline 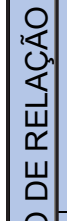 & 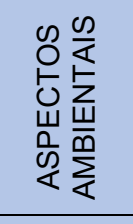 & & 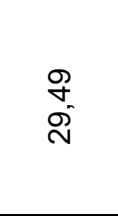 & 尺 & & D & $\nabla$ & $m$ \\
\hline $\begin{array}{l}\text { O } \\
\stackrel{2}{F} \\
\end{array}$ & 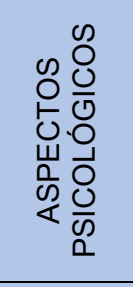 & 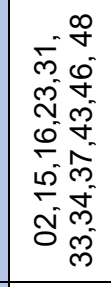 & 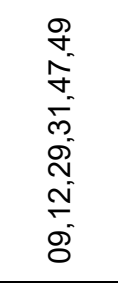 & 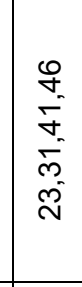 & & g & $\approx$ & $\neq$ \\
\hline & 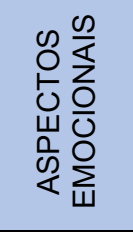 & 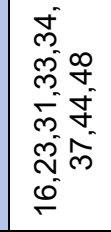 & 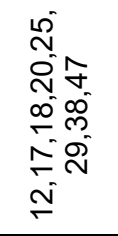 & 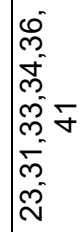 & $\mid \begin{array}{l}\bar{m} \\
\stackrel{\infty}{\infty}\end{array}$ & & $\stackrel{\sim}{\sim}$ & $\stackrel{2}{\circ}$ \\
\hline & 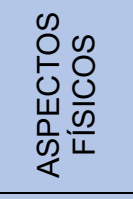 & 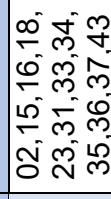 & 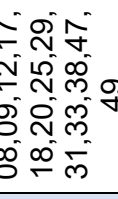 & 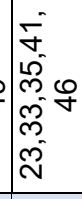 & 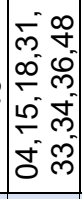 & 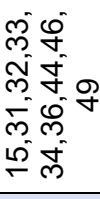 & f in & f \\
\hline & 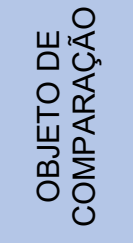 & 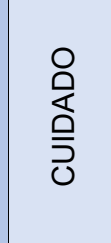 & 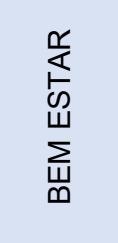 & $\stackrel{\nwarrow}{\stackrel{\Xi}{>}}$ & 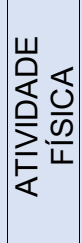 & 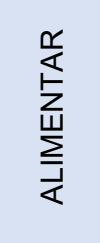 & 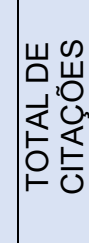 & \\
\hline
\end{tabular}


Figura 1. Análise de similitude utilizando o gráfico Kamaad-Kawa

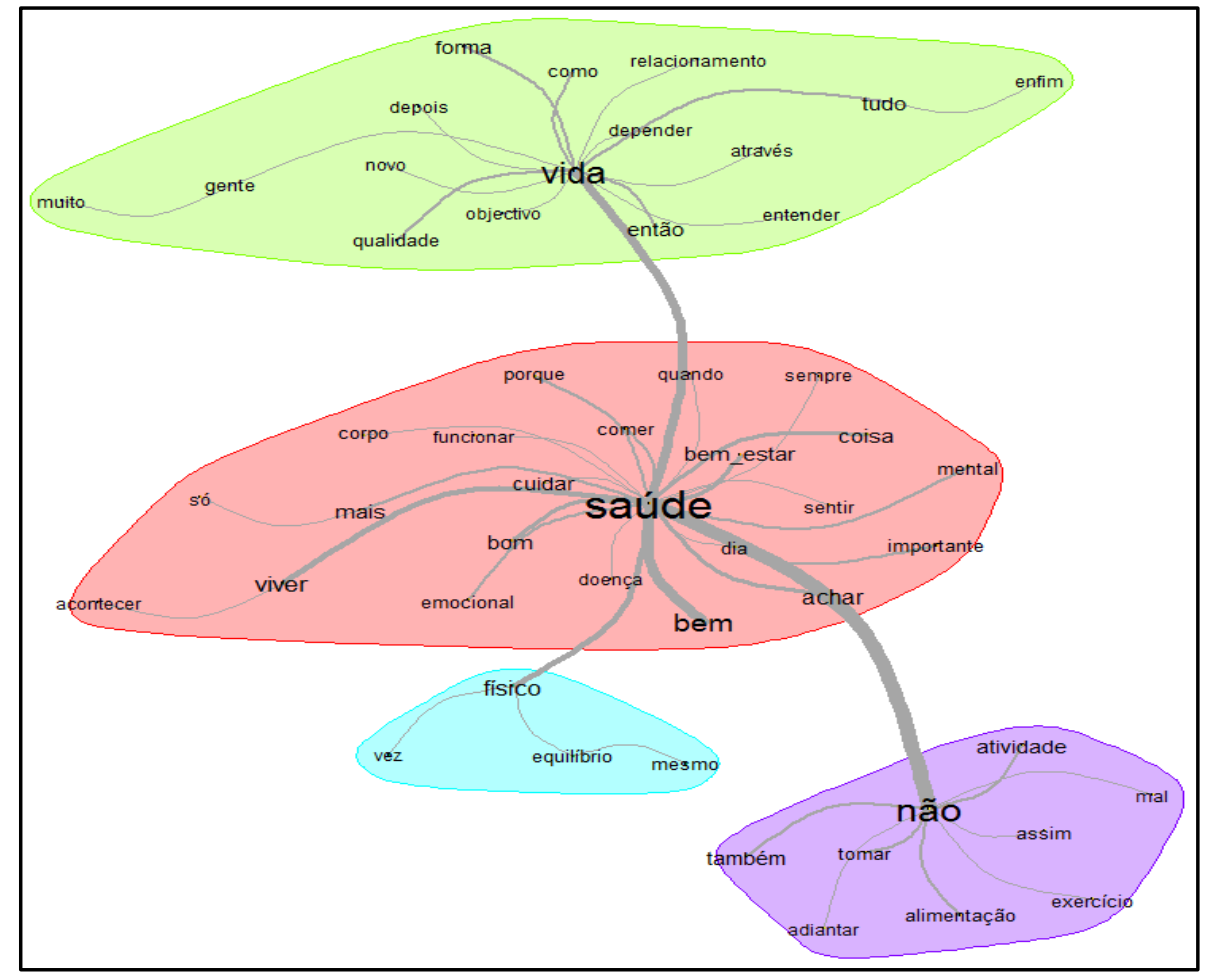

Figura 2. Nuvem de palavras da entrevista sobre saúde

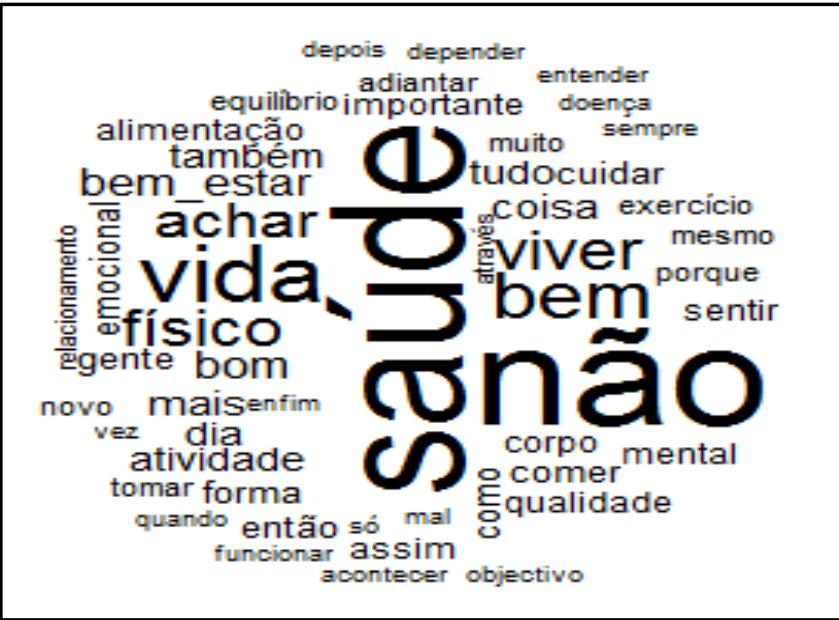


porquê estamos aqui, qual é o propósito existencial. Eu acho que é isto que me satisfaz, é ter este desejo, essa motivação de viver a cada dia através de objetivos e propósitos que eu entendo para a minha própria vida e no relacionamento com as pessoas."

Observa-se nas respostas dos idosos que a percepção de saúde está ligada a fatores externos (temas) que, na maioria das vezes, direciona os fatores internos (domínios), tornando cada um co-dependente do outro. Esses fatores, conduzem a uma linha de determinado conceito. No estudo de Menezes et al. ${ }^{14}$, que investigou a autopercepção de idosas com o envelhecimento, as participantes associaram seus conceitos à vários problemas relacionados aos aspectos físicos e individuais, que culminavam no declínio de aspectos que atingem a questão social como as doenças, a invalidez, o cansaço, a perda da independência e da autonomia, a acomodação e o desânimo com a vida ${ }^{14}$.

A Figura 1 apresenta graficamente a aplicação do corpus textual na ferramenta de análise de similitude. Pode-se perceber as ligações entre os temas principais, pela espessura das linhas e as famílias de cada tema. No centro está a família da palavra "saúde", demonstrando a importância deste tema, levando a duas outras ligações com linhas visivelmente espessas "vida" e "não", reflexo das respostas das participantes sobre a percepção de saúde.

Ao centro verifica-se o termo "saúde" que estabelece relações com sua família de palavras como "bem-estar", "emocional", "viver", "mental", "cuidar", "funcionar", "comer", "doença", "sentir" e "corpo", levando a crer que a percepção de saúde para as participantes envolve todos esses fatores e não está relacionada apenas a ausência de doença.

A Figura 1 apresenta uma nuvem de palavras extraída do mesmo processo de análise através do software IRaMuTeQ, de forma que são confirmadas as relações orbitais pela proximidade e pelo tamanho da palavra e graficamente é possível uma melhor visualização das falas dos participantes.

Sendo o termo "saúde" o que se destaca e estabelece uma ligação com o tema "vida", entende-se que a saúde é vida ou pode levar a ela, ou que a vida está diretamente relacionada à saúde. O tema de menor força no algoritmo é o "físico", corroborando em sua família de palavras a necessidade de estar em equilíbrio com as outras esferas já citadas.

O tema "não", neste contexto, remete em sua família de palavras ao que não leva a saúde, a ausência de algo que não está em equilíbrio: a má alimentação, a falta de exercícios e atividades, quer sejam elas atividades de vida diária ou cognitivas. Em outro contexto, a palavra "não" relaciona-se a não tomar medicamentos, não sentir dores, não sentir fadiga, não ter mal estar, não apresentar nenhum sinal e sintoma de doença.

Lindemann et al. ${ }^{15}$ verificaram em sua pesquisa que a probabilidade da autopercepção negativa da alimentação foi menor conforme o aumento da idade, pois para os idosos, a qualidade da alimentação, pode ter gerado uma diminuição da autopercepção negativa da alimentação ${ }^{17}$. Porém, os idosos deste presente estudo, não descartam o fato de que comer bem e ter uma má alimentação influenciam em sua percepção de saúde. O participante 16 afirma em sua resposta: "Saúde é a coisa mais importante no ser hu- 
mano (...) sempre se alimentar corretamente, comendo alimentação saudável, não abusar da bebida, cigarro e tudo aquilo que faz mal ao organismo."

O participante 17 afirma "não abusar da bebida" deixa implícito que a bebida pode ser consumida, porém, não se deve abusar do seu consumo. Krug et al. ${ }^{16}$ apresentaram em sua pesquisa que, a autopercepção de saúde positiva, foi menos prevalente nos idosos longevos sem sintomas depressivos e mais prevalente naqueles que consumiam álcool. No entanto, estes resultados podem ser questionados por alguns vieses. Um viés é a condição econômica, na qual pessoas com melhores rendas consomem mais álcool e podem acessar os serviços de saúde com mais frequência, reduzindo assim o impacto do álcool na saúde. Outro ponto, é que provavelmente os idosos que consomem álcool fazem isso porque estão com a saúde melhor e essa é a explicação mais provável para a associação encontrada entre o álcool e a autopercepção positiva de saúde, além dos laços sociais ${ }^{16}$.

A Figura 2 apresenta uma nuvem de palavras extraída do mesmo processo de análise por meio do software IRaMuTeQ, de forma que são confirmadas as relações orbitais pela proximidade, pelo tamanho da palavra e, graficamente, é possível uma melhor visualização das falas dos participantes, confirmando os temas de palavras já mencionados anteriormente "saúde", "vida", "não" e acrescentando o tema "viver bem".

A nuvem de palavras deixa explícito que saúde é vida, saúde é viver bem, saúde é não depender e ter autonomia nas suas escolhas, não tomar medicamento, é comer com qualidade, é um bom relacionamento com os outros e consigo mesmo, no sentido de autocuidado, levando a um bem-estar físico e emocional, é poder tomar suas próprias decisões. Gomes, Pereira e Abreu ${ }^{17}$ encontraram, em sua pesquisa, que a autopercepção de saúde dos idosos e os fatores associados a uma melhor percepção de saúde foram a satisfação com a relação com os amigos, não ter diabetes e nenhuma doença cardiovascular, a baixa frequência e a ausência dos problemas que dificultam os idosos de fazerem as coisas que precisam ou querem fazer ${ }^{17}$.

No estudo de Ribeiro et al. ${ }^{18}$ destacou-se a significância em relação à autopercepção de saúde negativa, nas variáveis de humor e de internação nos últimos seis meses. Os resultados mostraram que a prevalência de idosos com autopercepção negativa de saúde é 1,43 vez maior nos idosos que apresentaram tristeza ou desesperança, corroborando com o presente estudo ${ }^{19}$. O que retoma a uma das respostas, no qual a participante 2 afirma "Saúde é não ter que tomar medicamentos (...) ter autonomia de ir e vir aonde eu quero". Verifica-se que os resultados da Figura 1 e da Figura 2 dialogam entre si, reforçando a importância dos termos destacados com as respostas relacionadas aos temas, levando a crer que as respostas também estão vinculadas a autonomia e a perda funcional.

\section{CONCLUSÕES}

A percepção de saúde dos idosos, deste estudo, mostrou que estão ligadas a fatores externos (temas) que, na maioria das vezes, direciona os fatores internos (domínios), tornando cada um co-dependente do outro. Correlacionando com o ponto de vista dos idosos, percebe-se um tom de muita con- 
fiança e certeza do que é saúde para cada uma delas.

Verifica-se que o termo "saúde" estabelece relações com o "bem-estar", "emocional", "viver", "mental", "cuidar", "funcionar", "comer", "doença", "sentir" e "corpo", levando a crer que a percepção de saúde para as participantes envolve todos esses fatores e não está relacionado apenas a ausência de doença. Os resultados obtidos dialogam entre si, reforçando a importância dos termos destacados com as respostas relacionadas aos te-

\section{REFERÊNCIAS}

1. Instituto Brasileiro de Geografia e Estatística. Síntese de indicadores sociais: uma análise das condições de vida da população brasileira. IBGE. Rio de janeiro. 2015; p. 137

2. Papalia DE, Olds SW, Feldman RD. Desenvolvimento Humano. $8^{a}$ Edição. ed. Porto Alegre: Artmed Editora SA. 2006.

3. Coni N, Davison W, Webster S. O envelhecimento. São Paulo: Experimento. 1996.

4. Neri AL. Qualidade de vida no adulto maduro: interpretações teóricas e evidências de pesquisa. In: NERI, A. L. Qualidade de vida e idade madura. $2^{\mathrm{a}}$ Ed. ed. Campinas/ SP: Papirus. 1999; p. 9-56. Coleção Vivaidade.

5. Organização Mundial da Saúde. Relatório mundial de envelhecimento e saúde. 2015.

6. Nemes MC, Vagetti GC. Musicoterapia, saúde e idoso: uma revisão sistemática dos últimos 15 anos. In: Encontro Anual de Iniciação Científica da UNESPAR; 2015; Curitiba. Paraná: UNESPAR; 2015.

7. Gouveia OMR, Matos C, Schouten MJ. Social networks and quality of life of elderly persons: a review and critical analysis of literature. Rev Bras Geriatria e Gerontologia. 2016; 19 (6).

8. Scoralick-Lempke NN, Barbosa AJG, Mota MMPED. Efeitos de um Processo de Alfabetização em Informática na Cognição de Idosos. Psicologia: Reflexão e Crítica, Porto Alegre. 2012; 4(25).

9. Silveira MMD, Tavares GMS, Zuppa C, Portuguez MW, Filho IGS, Carli GA et al. A quality-of-life analysis of elderly computer workshop patrons. ConScientiae Saúde. 2013; 12(4).

10. Kreis RA, Alves VP, Cárdenas CJ, Karnikowski MGO et al. O impacto da informática na vida do idoso. Kairós, São Paulo; 2007, p. 153-168. mas, vinculando as respostas a autonomia, a perda funcional, ao bem-estar físico e mental.

Obtém-se deste estudo, sob o ponto de vista das idosas, que saúde é vida, e que o bem-estar físico e mental é vivenciado quando não existe dependência de outros e tem autonomia para suas escolhas. Outras características levantadas foram: não tomar medicamento, comer com qualidade, bom relacionamento com os outros e consigo mesmo, no sentido de autocuidado, e perspectivas de futuro.

11. Mazo G. Atividade física e qualidade de vida de mulheres idosas. Tese de Doutorado em Ciências do Desporto. Porto: Universidade do Porto; Faculdade de Ciências do Desporto e de Educação Física; 2003.

12. Associação Brasileira de Empresas de Pesquisa. Critério de classificação econômica do Brasil. São Paulo: ABEP 2018.

13. Bardin L. Análise de Conteúdo. São Paulo: Edições 70; 2016

14. Menezes JN, Tomaz BS, Pontes VF, Belchior LD. A Autopercepção de Idosas sobre o Processo de Envelhecimento. Estudos Interdisciplinares de Envelhecimento, Porto Alegre. 2016; 21(1):135 -148.

15. Lindemann IL, Barros KS, Mendoza-Sassi RA. Autopercepção da alimentação entre usuários da atenção básica de saúde e fatores associados. Rev Bahiana de Saúde Pública. 2017; 41(2):424-439.

16. Krug RR, Schineider IJC, Giehl MWC, Antes DL, Confortin $\mathrm{SC}$, Mazo GZ, et al. Fatores sociodemográficos, comportamentais e de saúde associados à autopercepção de saúde positiva de idosos longevos residentes em Florianópolis, Santa Catarina. Rev Bras Epidemiol. 2018; 21: e180004

17. Gomes MFS, Pereira SCL, Abreu MNS. Fatores associados à autopercepção de saúde dos idosos usuários dos restaurantes populares de Belo Horizonte. Ciência \& Saúde Coletiva. 2018; 23(11):4007-4019.

18. Ribeiro EG, Matozinhos FP, Guimarães GL, Couto AM Azevedo RS, Mendoza IYQ. Self-perceived health and clinicalfunctional vulnerability of the elderly in Belo Horizonte/Minas Gerais. Rev Bras Enferm [Internet]; 2018, 71(suppl 2):860-867.

\section{CORRESPONDÊNCIA}

Gislaine Cristina Vagetti

UNESPAR, Campus de Curitiba II

R. dos Funcionários, 1357 - Cabral,

Curitiba - PR, 80035-050

Email: gislainevagetti@hotmail.com 